Fundación

Miguel Lillo

Tucumán

Argentina

\title{
Sobre-veraneo de Calidris canutus rufa (Charadriiformes: Scolopacidae) en laguna La Picasa, Santa Fe, Argentina
}

\begin{abstract}
Over-summering of Calidris canutus rufa (Charadriiformes: Scolopacidae) in La Picasa Lake, Santa Fe, Argentina
\end{abstract}

\section{Martin Juárez}

Catamarca 908, Rufino, Santa Fe.

Correo electrónico: robertomartinj@yahoo.com.ar

\section{RESUMEN}

Calidris canutus rufa es una ave playera que tiene su área de reproducción en el Ártico canadiense, y ha sufrido una drástica declinación desde principios de este milenio, con tendencia decreciente de su tamaño poblacional. En el verano austral migra al sur de Sudamérica donde tiene una de las áreas principales de descanso no reproductivo en la Isla Grande de Tierra del Fuego. Es conocido que la especie sobre-veranea en las costas de la provincia de Buenos Aires, Argentina, sin reportes para el interior continental del país. A partir del avistamiento de Calidris canutus rufa en la laguna La Picasa, al sur de la provincia de Santa Fe, se realizaron muestreos semanales para registrar su presencia durante el invierno austral en este humedal. Se obtuvieron registros de la especie entre julio y septiembre de 2021 en la costa oeste de la laguna. Se trató de ejemplares con plumaje básico, con coloración gris cenicienta en dorso y blanca ventral, flancos barrados y patas verdosas. Este sería el primer registro de Calidris canutus rufa sobre-veraneando en laguna La Picasa, humedal del interior continental de Argentina, en el invierno austral 2021.

Palabras clave - Playero rojizo, humedal interior.

\begin{abstract}
Calidris canutus rufa is a shorebird that has its breeding area in the Canadian Arctic,and has suffered a drastic decline since the beginning of this millennium, with a decreasing trend in its population. In the austral summer it migrates to

Ref. bibliográfica: Juárez, M. 2021. "Sobre-veraneo de Calidris canutus rufa (Charadriiformes: Scolopacidae) en laguna La Picasa, Santa Fe, Argentina". Acta zoológica lilloana 65 (2): 253-260. doi: https://doi. org/10.30550/j.azl/2021.65.2/2021-10-18

> Recibido: 12 de septiembre 2021 - Aceptado: 18 de octubre 2021.

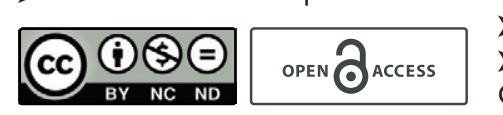

- URL de la revista: http://actazoologica.lillo.org.ar

- Esta obra está bajo una Licencia Creative Commons Atribución - No Comercial - Sin Obra Derivada 4.0 Internacional.
\end{abstract}


southern South America where it has one of the main non-reproductive rest area, in Isla Grande de Tierra del Fuego. It is known that the species over-summering on the coasts of the province of Buenos Aires, Argentina,without reports for the inland of the country. From the sighting of Calidris canutus rufa in La Picasa Lake, in the south of the province of Santa Fe, weekly samplings were carried out to record its presence during the southern winter in this wetland. Records of the species were obtained between July and September 2021 on the west coast of the lake. These were individuals with basic plumage, with ashy-gray coloration on the back and ventral white, barred flanks and greenish legs. Presented here is the first record of Calidris canutus rufa over-summering in La Picasa Lake, inland wetland of Argentina, in the southern winter 2021.

Keywords - Red Knot, inland wetland.

El Playero rojizo (Calidris canutus, Charadriiformes: Scolopacidae) es una ave cosmopolita que se reproduce en el Círculo Polar Ártico y pasa su estación no reproductiva en las costas de todos los continentes excepto la Antártida. Ha sufrido una drástica declinación desde principios de este milenio, con tendencia decreciente de su tamaño poblacional (Niles et al., 2008; Morrison, Ross y Niles, 2009); por tal motivo, en los últimos años está incluida dentro de las especies cercanas a la amenaza (NT) de la Lista Roja de la Unión Internacional para la Conservación de la Naturaleza (BirdLife International, 2018).

De las seis subespecies descriptas, Calidris canutus rufa Wilson A (1813), con sitio de reproducción en la tundra central canadiense entre los meses de junio y agosto, es la única que llega al sur de Sudamérica (Piersma y Davidson, 1992; Tomkovich, 2001). Sus principales áreas de descanso no reproductivo se ubican a lo largo de la costa oeste de Florida y sudeste de Estados Unidos, noroeste del Golfo de México, Maranhão en Brasil y la Isla Grande de Tierra del Fuego en el sur de Argentina y Chile (Niles et al., 2008; Andres et al., 2012). En Argentina su presencia se asocia principalmente a costas marinas y estuariales del litoral Atlántico (Harrington y Morrison, 1980).

Para esta especie se describe, además, el fenómeno de sobre-veraneo: aves migrantes boreales que no migran al norte y permanecen en el sur, lejos de sus áreas de cría, durante el verano boreal (Mc Neil, Diaz y Villeneuve, 1994). En Argentina, se reportaron Calidris canutus rufa sobre-veraneando en la costa de la provincia de Buenos Aires (Punta Rasa, Mar Chiquita y Claromecó), que incluyeron desde grupos pequeños a bandadas de 500 individuos (Blanco, Rodriguez Goñi y Pugnali, 1992; Blanco y Carbonell, 2001; Martínez Curci, 2016).

Se presenta aquí lo que sería el primer registro conocido de Calidris canutus rufa sobre-veraneando en el interior continental de Argentina, en la laguna La Picasa al sur de la provincia de Santa Fe en el invierno de 2021.

La laguna La Picasa es un extenso humedal ubicado en el sudoeste de la provincia de Santa Fe, entre los $34^{\circ} 16^{\circ}-34^{\circ} 26^{\prime} \mathrm{S}$ y los $62^{\circ} 08^{\circ}-62^{\circ} 22^{\prime} \mathrm{O}$. Tiene una superficie actual de 18000 ha. Pertenece a la región Humedales de la Pampa, subregión Lagunas 
salobres de la Pampa Interior (Benzaquen et al., 2017). Es un cuerpo de agua vinculado al paleocauce del Río Quinto, con sales de concentración variable, ubicado en un paisaje caracterizado por médanos arenosos aplanados que carecen de pendiente y de red fluvial, lo que permitió la formación de una cuenca endorreica (Bilenca y Miñarro, 2004). La zona ha sido destacada por la presencia de aves amenazadas y como sitio de importancia para la nidificación de aves vulnerables (Maugeri, Rey y Raparo, 2009; Mollo, Luna y Manassero, 2010;Juárez, 2021). Al momento de los muestreos la cota hidrométrica de la laguna estaba en baja (102 msnm) después del pico de 2017 (105,85 msnm, según datos del Instituto Nacional del Agua), dejando playas barrosas de hasta $100 \mathrm{~m}$ de ancho en algunos puntos de la costa oeste y sur.

A partir del avistamiento de Calidris canutus rufa el 3 de julio de 2021 en laguna La Picasa, se realizaron campañas de muestreo buscando específicamente esta especie para registrar su presencia durante los meses de julio a septiembre de 2021. El recorrido se realizó en la costa oeste de la laguna, a pie, desde las vías del ferrocarril al norte hasta el canal aliviador al sur (Fig. 1), completando una distancia de $8 \mathrm{~km}$. En total se realizaron 10 muestreos, todos por la mañana de 08:00 a 12:00, cada 7 a 8 días. En condiciones climáticas adversas (lluvia intensa o viento fuerte) no se realizaron muestreos.

El avistamiento se realizó utilizando binoculares 10x. Para la identificación de la especie se siguió la Guía de aves de Narosky e Yzurieta (2010).

Entre el 3 de julio y el 4 de septiembre de 2021 se obtuvieron 12 registros de Calidris canutus rufa. De los 10 muestreos, en cinco la especie estuvo ausente (Tabla 1, Fig. 1, 2 y 3). En todos los casos se trató de ejemplares con plumaje básico, con coloración gris cenicienta en dorso y blanca ventral, flancos barrados y patas verdosas. Se los encontró forrajeando o en descanso en las costas de la laguna, junto a otras especies como Himantopus mexicanus, Charadrius collaris, Charadrius modestus y Limosa haemastica. No se observaron actitudes de agresividad o competencia entre las especies. La máxima cantidad de Calidris canutus rufa observada por muestreo individual fue de tres individuos.

Tabla 1. Registros de Calidris canutus rufa en laguna La Picasa. Abundancia: cantidad de individuos observados.

Table 1. Records of Calidris canutus rufa in La Picasa Lake. Abundance: number of individuals observed.

\begin{tabular}{|c|c|c|}
\hline Fecha de los muestreos & Abundancia & Registro \\
\hline $03-07-21$ & 3 & Fig. 2: https://ebird.org/argentina/checklist/S91199611 \\
\hline $11-07-21$ & - & \\
\hline $17-07-21$ & 3 & Fig. 3 A \\
\hline $24-01-21$ & - & https://ebird.org/argentina/checklist/S92660035 \\
\hline $31-07-21$ & 2 & \\
\hline $08-08-21$ & - & Fig. 3 B \\
\hline $14-08-21$ & - & \\
\hline $21-08-21$ & 3 & Fig. 3 C \\
\hline $29-08-21$ & - & 1 \\
\hline $04-09-21$ & &
\end{tabular}




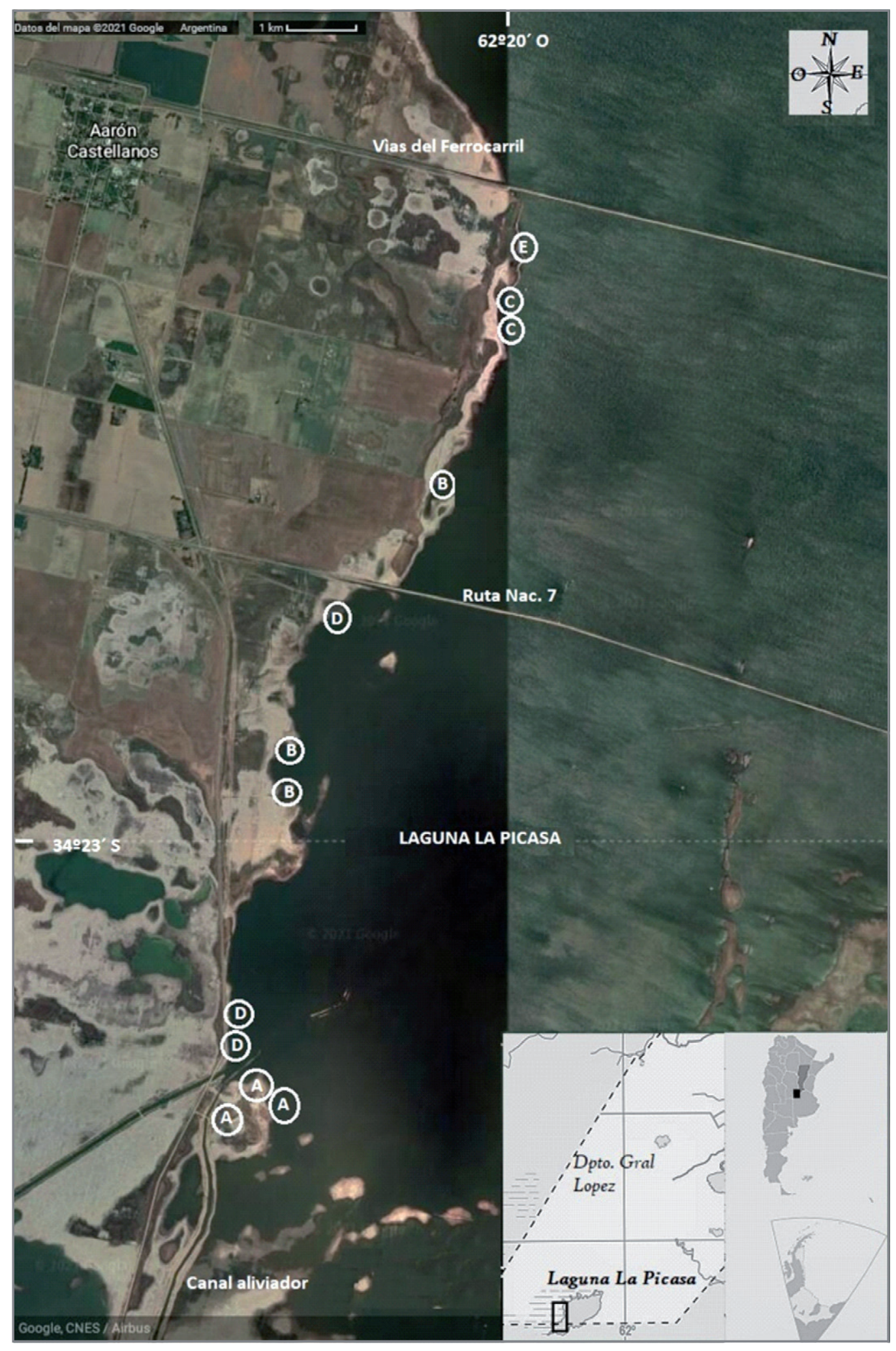

Fig. 1. Ubicación de los registros de Calidris canutus rufa en la costa oeste de laguna La Picasa: A) 03-07-21; B) 17-07-21; C) 31-07-21; D) 21-08-21; E) 04-09-21. Mapa base: Google Earth, 2021. Mapa miniatura: Instituto Geográfico Nacional, 2020.

Fig. 1. Location of the records of Calidris canutus rufa on the west coast of La Picasa Lake: A) 0703-21; B) 07-17-21; C) 07-31-21; D) 08-21-21; E) 09-04-21. Basemap: Google Earth, 2021. Miniature map: Instituto Geográfico Nacional, 2020. 


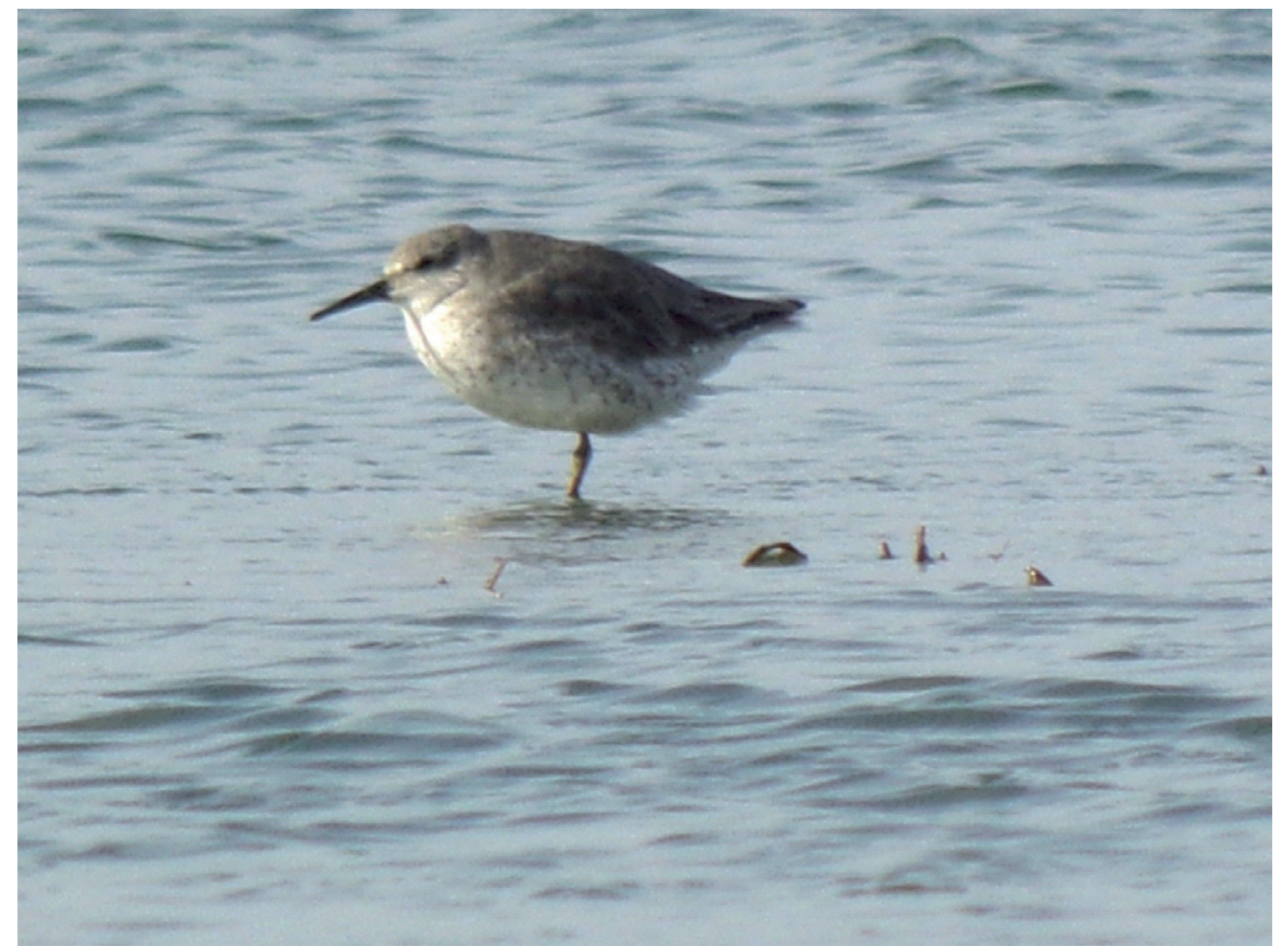

Fig. 2. Ejemplar de Calidris canutus rufa observado el 3 de julio de 2021 en laguna La Picasa. Foto: Martín Juárez.

Fig. 2. Specimen of Calidris canutus rufa observed on July 3, 2021 in La Picasa Lake. Photo: Martín Juárez.
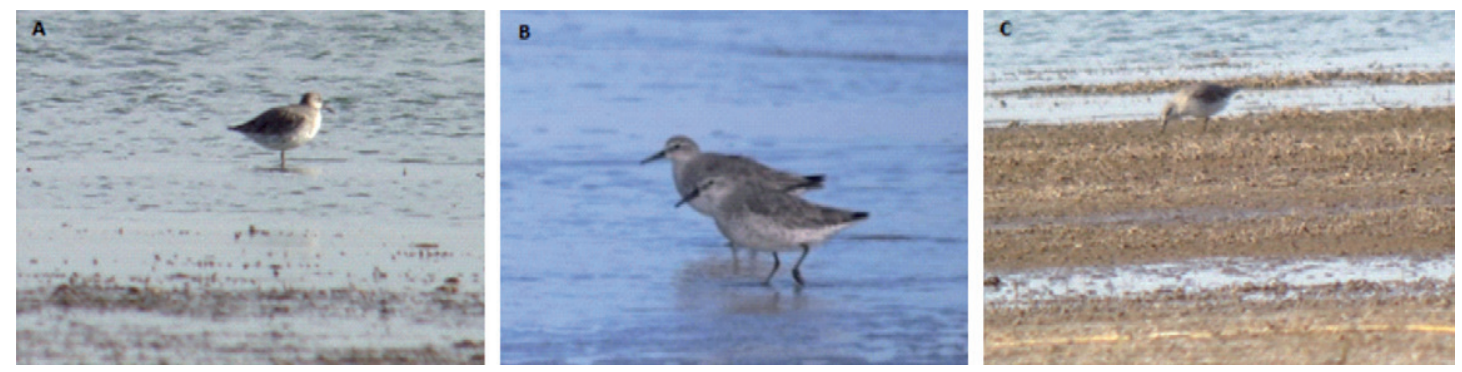

Fig. 3. Registros fotográficos de Calidris canutus rufa en laguna La Picasa: A) 17-07-21, B) 21-08-21, C) 04-09-21. Fotos: Martín Juárez.

Fig. 3. Photographic records of Calidris canutus rufa in La Picasa Lake: A) 07-17-21, B) 08-21-21, C) 09-04-21. Photos: Martín Juárez.

En Argentina, hay pocos registros de la presencia de esta especie en el interior continental.

En la primavera austral (época de migración hacia el sur) hay reportes en laguna Mar Chiquita, Córdoba (registros de 1 o 2 individuos entre 1991 y 2018; eBird Argentina: mapa de distribución, 2021); en el Parque Nacional El Palmar, Entre Ríos (un individuo errante en 1991; Sarquis, Alonso, Giraudo y Berduc, 2017) y, 
recientemente, en laguna Paiva, Santa Fe (un individuo; eBird Argentina: mapa de distribución, 2021); en laguna El Cristal, Santa Fe (un individuo; eBird Argentina: mapa de distribución, 2021) y en la isla Cambacuá sobre el río Uruguay, Entre Ríos (un individuo; EcoRegistros, 2021).

En el otoño austral (época de migración hacia el norte) hay reportes en paso de la Cina, Santiago del Estero (3 individuos en 1986; Coria et al., 2021); laguna Mar Chiquita, Córdoba (frecuencia escasa; Torres y Michelutti, 2006); y laguna de Melincué, Santa Fe (conteos de 128, 18 y 5 individuos en 1999, 2007 y 2008 respectivamente; Romano, Barberis, Luppi y Pagano, 2015).

No se conocen reportes para el interior continental del país en otras épocas del año. Por lo tanto, el aquí presentado sería el primer registro de sobre-veraneo de Calidris canutus rufa en un humedal del interior continental de Argentina en el invierno austral. Si bien en el presente trabajo se obtuvieron 12 registros de la especie, por la falta de marcación, no se puede descartar que se haya tratado de un pequeño número de individuos que hayan cambiado su ubicación durante los muestreos. De todos modos, se demuestra la presencia de la especie en laguna La Picasa y su permanencia durante los meses de julio a septiembre de 2021.

Todo esto permitiría confirmar la importancia de este humedal, sin protección formal aún, para la conservación de chorlos y playeros migrantes; y mostrar la posibilidad de que la laguna La Picasa sea un área de sobre-veraneo para Calidris canutus rufa.

\section{AGRADECIMIENTOS}

Agradezco a Alejandro Spianamonte por la colaboración en el trabajo en terreno y a Natalia Martínez Curci por compartir recorridos por laguna La Picasa.

\section{FINANCIAMIENTO}

El trabajo fue financiado con recursos propios del autor.

\section{PARTICIPACIÓN}

Tanto el trabajo de campo como la redacción del manuscrito estuvieron a cargo del autor, con colaboración de A. Spianamonte en las campañas.

\section{CONFLITOS DE INTERÉS}

El autor declara que no presenta conflictos de interés de ningún tipo. 


\section{LITERATURA CITADA}

Andres, B.A., Smith, P.A., Morrison, R.I.G., Brown, S.C., Gratto-Trevor, C.L.\& Friis, C.A. (2012). Population estimates of North American shorebirds, 2012. Wader Study Group Bull., 119 (3), 178-194.

Benzaquen, L., Blanco, D.E., Bo, R., Kandus, P., Lingua, G., Minotti, P. \& Quintana, R. (2017). Regiones de Humedales de la Argentina. Ministerio de Ambiente y Desarrollo Sustentable. Fundación Humedales / Wetlands International. Universidad Nacional de San Martín y Universidad de Buenos Aires.

Bilenca, D.N. \& Miñarro, F.O. (2004). Identificación de áreas valiosas de pastizal (AVPs) en las pampas y campos de Argentina, Uruguay y sur de Brasil. Fundación Vida Silvestre Argentina. Buenos Aires.

BirdLife International (2018). Calidris Canutus. The IUCN Red List of Threatened Species: 2018. Disponible en https://www.iucn redlist.org/species/22693363/132285482 Accedido el 10-07-21

Blanco, D., Rodriguez Goñi, H. \& Pugnali, G. (1992). La importancia de Punta Rasa, Buenos Aires, en la migración del Chorlo rojizo (Calidris canutus). Hornero, 13, 203-206.

Blanco, D.E. \& Carbonell, M. (2001). The Neotropical Waterbird Census: the first 10 years: 1990-1999. Wetlands International \& Ducks Ulimited. Inc: Memphis, Estados Unidos.

Coria, O.R., Quiroga, O.B., Navarro, J.L., Heredia, J., Torres, R.\& Lima, J. (2021). Lista actualizada de las aves de Santiago del Estero, Argentina. Acta Zoológica Lilloana, 65 (1), 42-143.

EcoRegistros (2021). Playero rojizo (Calidris canutus). Ficha de la especie. Accedido de http://www.ecoregistros.org el 15-10-21.

eBird Argentina (2021). Mapa de distribución del Playero rojizo (Calidris canutus). Disponible en https://ebird.org/argentina/species/redkno/AR, accedido el 1510-21.

Google Earth (2021). Mapa satelital laguna La Picasa. Disponible en https://www. google.com/earth/studio/, accedido el 10-09-21.

Harrington, B.A. \& Morrison, R.I.G. (1980). Notes on the wintering areas of Red Knot Calidris canutus rufa in Argentina, South America. Wader Study Group Bull., 28, 40-42.

Instituto Geográfico Nacional (IGN). (2021). Mapa Satelital de la provincia de Santa Fe, República Argentina. Mapa físico-político. Descargado de https://www.ign. gob.ar, accedido el 10-09-2021.

Juárez, M. (2021). Variación estacional de la riqueza y frecuencia relativa de aves acuáticas de la laguna "La Picasa", Santa Fe, Argentina. Nuestras Aves, 66, 35-41.

McNeil, R., Diaz, M.T. \& Villenueve, A. (1994).The mystery of shorebird oversummering: a new hypothesis. Ardea, 82, 143-152.

Martínez Curci, N.S. (2016). Ecología de aves playeras migratorias durante la invernada, migración y sobre veraneo en Bahía Samborombón, Buenos Aires, Argentina. Tesis Doctoral. Facultad de Ciencia Exactas y Naturales. Universidad de Buenos Aires. 
Maugeri, F.G., Rey, L. \& Raparo, A. (2009). Laguna La Picasa, Santa Fe, como sitio de importancia para la nidificación de Phoenicopterus chilensis. Libro de resúmenes, XIII Reunión Argentina de Ornitología. Tucumán.

Mollo, H., Luna, H. \& Manassero, M. (2010). Importancia ornitológica del sector santafesino de la cuenca de la laguna La Picasa. Biológica, 12, 65-68.

Morrison, R.I.G., Ross, R.K. \& Niles, L.J. (2009). Declines in wintering populations of Red Knot in Southern South America. The Condor, 106 (1), 60-70.

Narosky, T. \& Yzurieta, D. (2010). Aves de Argentina y Uruguay: Guía de identificación. Vazquez Mazzini Editores. Buenos Aires.

Niles, L.J., Sitters, S.H.P., Dey, A.D., Atkinson, P.W., Baker, A.J., Bennett, K.A., Carmona, R., Clark, K.E., Espoz, C., Gonzalez, P.M., Harrington, B.A., Hernandez, D.E., Kalasz, K.Z., Lathrop, R.G., Matus, R.N., Minton, C.D.T., Morrison, R.I.G., Peck, M.K., Pittis, W., Rovinson, R.A.\& Serrano, I.L. (2008). Status of the Red Knot (Calidris canutus rufa) in the Western Hemisphere. Studies in Avian Biology, 36, 1-185.

Piersma, T. \& Davidson, N.C. (1992). The migrations and annual cycles of five subspecies of Knots in perspective. Wader Study Group Bull. 64, Sup, 187-197.

Romano, M., Barberis, I.M.,Luppi, M. \& Pagano, F. (2015). Non-passerine birds from Laguna Melincué Ramsar Site, Santa Fe province, Argentina. Check List The journal of biodiversity data, 11 (6), article 1799.

Sarquis, J.A., Alonso, J., Giraudo, A.R. \& Berduc, A. (2017). First records of Calidris canutus Linnaeus, 1758 (Charadriiformes, Scolopacidae) and Trogon surrucuraVieillot, 1817 (Trogonigormes, Trogonidae) for Entre Rios province (Argentina), and noteworthy reports of other birds in protected areas. Checklist, 13 (6), 1067-1073.

Tomkovich,P.S. (2001). A new subspecies Red Knot Calidris canutus from the New Siberian Islands. Bulletin British Ornithologists' Club, 121, 257-263.

Torres, R. \& Michelutti, P. (2006). Aves acuáticas. En: Bañados del Río Dulce y laguna Mar Chiquita (Córdoba, Argentina). Ed. Bucher E.H., 237-249. Academia Nacional de Ciencias Córdoba. 